\title{
Methods and efficacy of extracellular vesicles derived from mesenchymal stromal cells in animal models of disease: a preclinical systematic review protocol
}

\author{
Alvin Tieu ${ }^{1,2,3}$, Mitchell Slobodian², Dean A. Fergusson ${ }^{2,4,5}$, Joshua Montroy², Dylan Burger ${ }^{1,6,7}$, \\ Duncan J. Stewart ${ }^{1,3,4}$, Risa Shorr ${ }^{8}$, David S. Allan ${ }^{2,3,4^{*}}$ (D) and Manoj M. Lalu1, 1,3,4,9* $^{*}$
}

\begin{abstract}
Background: Over the past decade, mesenchymal stromal cells have been increasingly investigated for their therapeutic potential in several different illnesses. However, cell therapy can be limited by potentially serious adverse events including cell embolus formation and tumorigenesis. Importantly, the protective effects of mesenchymal stromal cells are largely mediated by paracrine mechanisms including release of extracellular vesicles. This systematic review intends to synthesize the current knowledge of mesenchymal stromal cell-derived extracellular vesicles as a therapeutic option for preclinical models of disease, inflammation, or injury.

Methods: A systematic literature search of MEDLINE, Embase, and BIOSIS databases will be conducted. Interventional preclinical in vivo studies using extracellular vesicles derived from any tissue source of mesenchymal stromal cells will be included. Studies will be screened by abstract, and full-text by two independent reviewers. Eligible studies will undergo data extraction with subcategorization into domains based on disease. Methods utilized for extracellular vesicle characterization and isolation will be collected, as well as information on interventional traits, such as tissue source of mesenchymal stromal cells, dosage regimen, and vesicle modifications. Reported outcomes will be collected to determine which diseases studied may be impacted most from treatment with mesenchymal stromal cell-derived extracellular vesicles.

Discussion: This systematic review will summarize preclinical studies investigating the therapeutic efficacy of both small and large extracellular vesicles derived by mesenchymal stromal cells. Extracellular vesicles represent a possibility to harness the benefits of mesenchymal stromal cells with added benefits of reduced manufacturing costs and an improved safety profile. Hence, there has been an exponential increase in interest for developing this cell-free therapy with hundreds of preclinical studies published to date. However, a vast amount of heterogeneity between groups relates to methods of extracellular vesicle isolation, characterization, and study design. This review will capture this heterogeneity and identify the most commonly used and optimal approaches to evaluate mesenchymal stromal cell-derived extracellular vesicle treatment. A meta-analysis of outcomes within each disease domain will help elucidate which fields of research demonstrate promise for developing extracellular vesicles as a novel cell-free therapy. Summarizing this robust information on extracellular vesicles as an intervention can provide (Continued on next page)
\end{abstract}

\footnotetext{
* Correspondence: daallan@toh.ca; mlalu@toh.ca

${ }^{2}$ Clinical Epidemiology Program, BLUEPRINT Translational Research Group,

Ottawa Hospital Research Institute, Ottawa, Canada

'Department of Cellular and Molecular Medicine, University of Ottawa,

Ottawa, Canada

Full list of author information is available at the end of the article
}

(c) The Author(s). 2019 Open Access This article is distributed under the terms of the Creative Commons Attribution 4.0 International License (http://creativecommons.org/licenses/by/4.0/), which permits unrestricted use, distribution, and reproduction in any medium, provided you give appropriate credit to the original author(s) and the source, provide a link to the Creative Commons license, and indicate if changes were made. The Creative Commons Public Domain Dedication waiver (http://creativecommons.org/publicdomain/zero/1.0/) applies to the data made available in this article, unless otherwise stated. 
(Continued from previous page)

guidance for designing preclinical studies with hopes of future clinical translation.

Keywords: Mesenchymal stromal cells, Mesenchymal stem cells, Exosomes, Microvesicles, Extracellular vesicles, Systematic review protocol, Preclinical

\section{Background}

Mesenchymal stromal cells represent a subset of adult stem-like cells which have been extensively investigated for therapeutic potential in a variety of disease states. Mesenchymal stromal cells have been isolated from numerous tissue sources including adipose tissue, umbilical cord, and synovial membrane [1], and they can be derived from induced pluripotent stem cells [2]. Their multilineage differentiation potential, in vitro expansive capacity, and ability for immunomodulation has led to nearly 500 clinical trials [3], including studies of septic shock [4], acute respiratory distress syndrome [5], and ischemic heart disease [6]. In particular, mesenchymal stromal cells have received approval as a therapy to treat graft versus host disease in New Zealand, Canada, and Japan [7, 8]. Cell therapies may be limited by potentially serious adverse events of cell embolus and tumor formation [9]. Thus, there is immense interest in harnessing the therapeutic effects of mesenchymal stromal cells in ways that avoid potential harms.

Mesenchymal stromal cells' effects are largely mediated by paracrine mechanisms, and so mesenchymal stromal cell-derived extracellular vesicles may represent a safer cell-free alternative to whole cell injections. Extracellular vesicles contain biologically active factors and signaling molecules to facilitate intercellular communication. With opportunities for diminished manufacturing and storage costs for extracellular vesicles, they can also be produced as a more readily available "offthe-shelf" product, as extracellular vesicles are more stable than whole cells when cryogenically frozen. Research into the development of cell-free therapies has focused primarily on two types of extracellular vesicles, medium-large vesicles known as microvesicles, and small extracellular vesicles, which include exosomes.

Microvesicles and exosomes are subsets of extracellular vesicles that range from $150-1000$ and $30-150 \mathrm{~nm}$ in diameter, respectively [10] (Fig. 1). Both microvesicles and exosomes are prolifically produced by mesenchymal stromal cells [11] and are believed to carry bioactive cargo, such as microRNA, transcription factors, growth factors, and other regulatory proteins that help mediate their potentially efficacious properties [12]. Extracellular vesicles may serve as ideal vesicles for encapsulating therapeutic products, protect these products from degradation, and aid their delivery to specific target tissues. The opportunity to introduce or manipulate the expression of contents inside extracellular vesicles (e.g., through overexpression of protective microRNAs) has created an intriguing new domain of regenerative medicine.

The systematic review stemming from this protocol will synthesize current evidence on mesenchymal stromal cellderived microvesicles and exosomes as therapeutic options in preclinical models of disease, inflammation, or injury. A scoping review previously published by our group systematically reviewed preclinical data on extracellular vesicles up to 2013 and found that sixteen of seventeen preclinical studies demonstrated therapeutic benefits associated with administration of mesenchymal stromal cell-derived extracellular vesicles [13]. Subsequently, the terminology surrounding extracellular vesicle research has matured, and interest in studying microvesicles and exosomes has increased exponentially, necessitating a re-evaluation of this body of evidence.

\section{Research objectives}

We will review in vivo preclinical studies evaluating mesenchymal stromal cell-derived extracellular vesicles as an intervention for any animal model of disease or injury. Our primary objective will assess the methodology being utilized in these animal studies including (1) interventional characteristics, (2) methods of extracellular vesicle enrichment and characterization, and (3) experimental design. Given the expected heterogeneity of methods used (e.g., cell sources, isolation techniques) and outcomes assessed across various disease domains, a synthesis of these issues is needed to provide context for any subsequent meta-analysis. For our secondary objective, studies will be categorized by disease domains with subsequent meta-analyses of study outcomes to determine the therapeutic efficacy of mesenchymal stromal cell-derived extracellular vesicles in different conditions. Results of this analysis will help guide future preclinical studies of extracellular vesicles and inform whether possible human participant studies are feasible.

\section{Methods and design \\ Review team}

The team members for this systematic review encompass researchers across different scientific disciplines. The team has expertise in systematic reviews (DAF, MML, JM, DSA), basic science studies investigating novel cell therapies (AT, MML, DJS, DSA), and translational cell therapy trials (DJS, DAF) $[6,14,15]$. As well, DB participated in the development of the recently published 


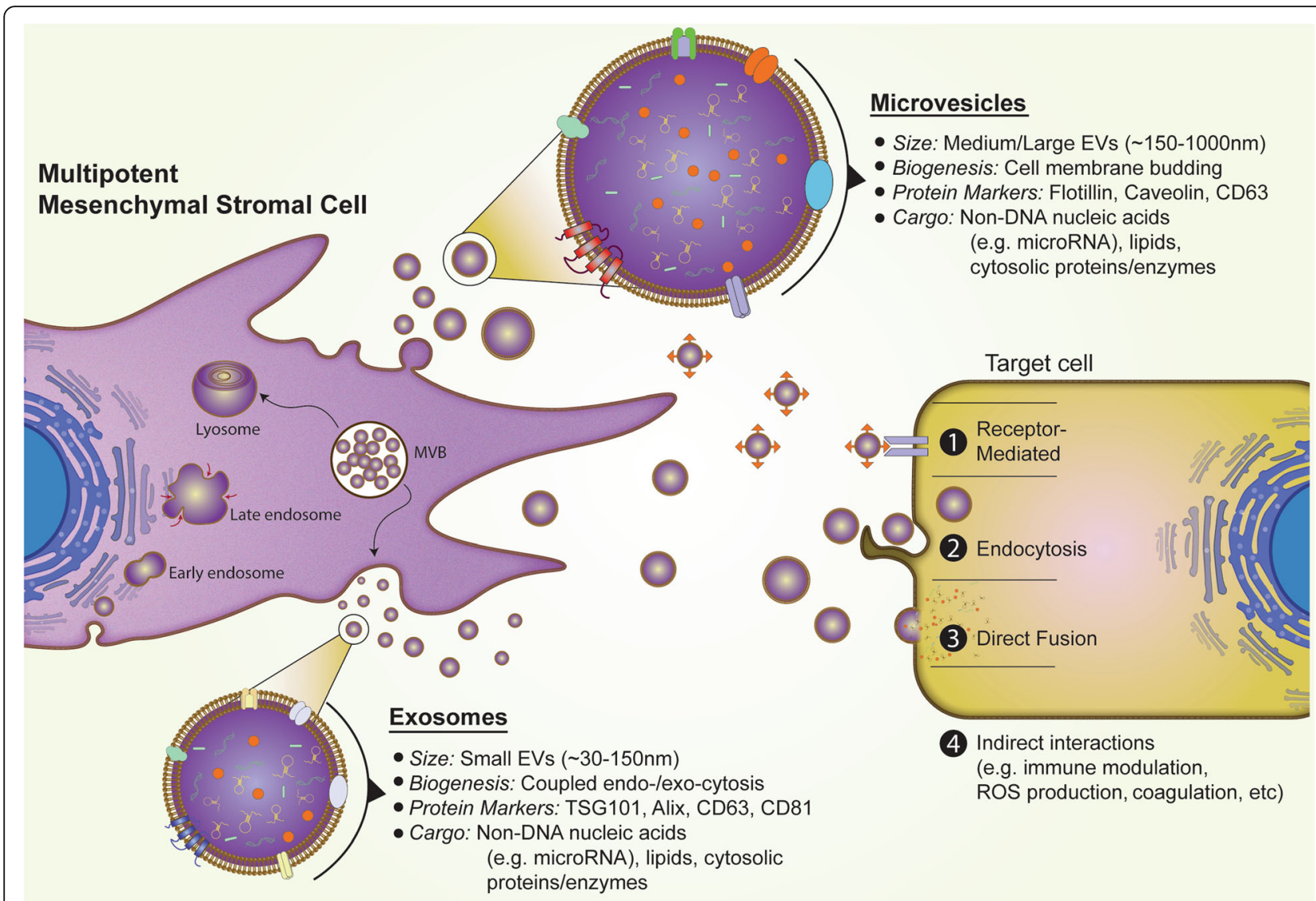

Fig. 1 Overview of extracellular vesicles derived from mesenchymal stromal cells. Size, biogenesis, protein markers, and vesicular cargo for both exosomes and microvesicles are described. Potential mechanisms by which extracellular vesicles communicate with target cells include receptor-mediated interactions, cellular endocytosis, direct fusion with cellular membrane, and indirect interactions such as immune modulation, reactive oxygen species production, and coagulation. MVB, multivesicular body; TSG101, tumor susceptibility gene $101 ; R O S$, reactive oxygen species

Minimal Information for Studies of Extracellular Vesicles 2018 (MISEV2018) position statement and provides considerable experience in the field of extracellular vesicle research [16]. The student leads for this project (AT and MS) have experience in both basic science and clinical epidemiology and will be the two primary reviewers for article selection and data extraction.

\section{Protocol}

This systematic review protocol is reported in accordance with the Preferred Reporting Items for Systematic reviews and Meta-Analysis Protocol (PRIMSA-P) reporting guidelines (Additional file 1) [17]. A summary of the protocol has been registered at the International Prospective Registry of Systematic Reviews (PROSPERO CRD42019123918).

\section{Eligibility criteria}

\section{Population}

Inclusion-in vivo preclinical animal models of disease, organ injury, tumor growth, or altered immune response.
Exclusion-ex vivo, in vitro, and invertebrate animal models will be excluded.

\section{Intervention}

Inclusion - mesenchymal stromal cell-derived extracellular vesicles including microvesicles $(150-1000 \mathrm{~nm})$ and exosomes $(30-150 \mathrm{~nm})$. Extracellular vesicles can be derived from xenogeneic, syngeneic, or allogeneic mesenchymal stromal cells from any tissue source. All routes of intervention administration will be considered, including direct tissue, intravenous, and intra-arterial injections. Experiments involving pre-treatment of mesenchymal stromal cells, co-treatment, and/or genetic manipulation (e.g., over- or under-expression of genes) before extracellular vesicle isolation will be classified as "modifications" for subgroup analysis. Exclusion-studies where extracellular vesicles are not directly administered to animals as a therapy (e.g., used as a pre-treatment for other interventions) or investigated only for their biodistribution will be excluded. 


\section{Comparators}

Studies with any comparator (e.g., vehicle control, placebo, mesenchymal stromal cells, and fibroblast microvesicles) will be considered.

\section{Outcomes}

Inclusion-studies that provide information regarding intervention characteristics, methodologic approaches and experimental design parameters utilized in studies will be included. For the purposes of a potential quantitative synthesis and meta-analysis, we will extract each study's primary outcome. If a primary outcome has not been outlined in the study, expert opinion or consensus statements will be sought to determine which in vivo outcome may be most clinically relevant for diseases treated.

\section{Study design}

Inclusion-interventional in vivo studies with a comparatorcontrol will be included. Studies may be randomized, pseudo-randomized, or non-randomized. Exclusion-unpublished gray literature, abstracts, review articles, editorials, and letters will be excluded. Single arm interventional studies will also be excluded.

\section{Data sources}

We will search Ovid MEDLINE, Ovid MEDLINE ${ }^{\bullet}$ InProcess \& Other Non-Indexed Citations, Embase Classic + Embase, and BIOSIS from inception to present day. In addition, hand searches of the bibliographies of included articles and relevant reviews will be performed.

\section{Search strategy}

The search strategies (Additional file 2) to be used for this review will be generated by a health sciences librarian (RS) with experience designing systematic literature searches. A second information specialist with no association to the project will also review the strategy using Peer Review of Electronic Search Strategies (PRESS) before the final search procedure is executed $[18,19]$. The search strategy will utilize both calculated vocabulary and $\mathrm{MeSH}$ terms (e.g., mesenchymal stem cells, mesenchymal stromal cells, extracellular vesicles, exosomes, and microvesicles), acronyms (e.g., MSCs, microvesicles, and extracellular vesicles), and $\mathrm{MeSH}$ terms with alterations as required per database. Our previous search strategy published in 2015 was updated to reflect evolving terminology in the extracellular vesicle field [13]. Preclinical filters will also be applied to improve search efficiency [20-22]. No search restrictions will be created for language or publication date.

\section{Study records}

\section{Data management}

Search results will be uploaded to Distiller Systematic Review Software (DistillerSR, Evidence Partners, Ottawa,
Canada). DistillerSR is an online software that allows for improved transparency, reproducibility, and accessibility for literature review.

\section{Selection process}

Two reviewers (AT, MS) will independently screen titles and abstracts from search results using the predefined inclusion criteria outlined above. A calibration test involving sets of 10 studies will be performed (AT, MS) to refine the screening question and ensure high inter-rater correlation (kappa $>0.8$ ), prior to formally commencing the screening process. For all titles that appear to meet the inclusion criteria or where there is any uncertainty, we will obtain the full-text article. Two reviewers (AT, MS) will assess the eligibility of full-text articles. A calibration test involving a set of 10 studies will be performed (AT, MS) to ensure high inter-rater correlation. After every calibration test, the entire review team will be consulted to resolve any issues concerning full-text inclusion. After refining the full-text screening criteria, a formal screening process (AT, MS) will be commenced. Any disagreement will be resolved through discussion with a third-party member (MML, DSA). Reasons for study exclusion at this level will be recorded. There will be no restrictions on language. Articles of non-English text will be translated by native speakers at the Ottawa Methods Centre.

\section{Data collection process}

A standardized data extraction form will be designed by the review team and uploaded to DistillerSR. Information will be extracted independently and in duplicate from each eligible study (AT, MS). Calibration exercises of 10 studies will be conducted, and the review team will be consulted to refine the data extraction form. After commencing formal data abstraction, any disagreements will be first discussed between two independent reviewers (AT, MS). If no resolution can be made, a thirdparty team member will be consulted (MML).

\section{Data items}

Broad categories of data items include methods of vesicle separation and characterization (Table 1), intervention details (e.g., tissue type of mesenchymal stromal cell, dose, route of administration, and timing of administration) (Table 2), study characteristics (e.g., study design, population of species, model of disease) (Table 3), and preclinical outcomes.

\section{Risk of bias assessment}

For each included study, risk of bias assessments will be carried out by two independent reviewers (AT, MS) using the Systematic Review Centre for Laboratory animal Experimentation (SYRCLE) risk of bias tool [23]. The SYRCLE tool was adapted from the Cochrane Risk of Bias Tool to assess the 
Table 1 Isolating and characterizing mesenchymal stem cellderived extracellular vesicles

\begin{tabular}{|c|c|}
\hline Question of interest & Example answers \\
\hline \multirow[t]{6}{*}{ Terminology used } & Extracellular vesicles \\
\hline & Exosomes \\
\hline & Microvesicles \\
\hline & Small EVs \\
\hline & Large EVs \\
\hline & Microparticles \\
\hline \multirow[t]{4}{*}{ Size of EVs } & Small EVs (30-150 nm) \\
\hline & Large EVs (150-1000 nm) \\
\hline & Both used together \\
\hline & Both used separately \\
\hline \multirow[t]{3}{*}{ Method of EV enrichment } & Ultracentrifugation \\
\hline & Isolation kit \\
\hline & Tangential flow filtration \\
\hline \multirow[t]{9}{*}{ Method of EV characterization } & Nanoparticle tracking analysis (size) \\
\hline & Dynamic light scattering (size) \\
\hline & Tunable resistive pulse sensing (size) \\
\hline & Western blot (protein content) \\
\hline & Flow cytometry (protein content) \\
\hline & ELISA (protein content) \\
\hline & Proteomics (protein content) \\
\hline & Electron microscopy (morphology) \\
\hline & Bradford/BCA assay \\
\hline \multirow[t]{5}{*}{ Protein markers used } & CD63 (seen in many EVs) \\
\hline & CD9 (seen in many EVs) \\
\hline & CD81 (small EV) \\
\hline & TSG101 (small EV) \\
\hline & Other (specify) \\
\hline \multirow[t]{2}{*}{ Negative protein markers } & Calnexin \\
\hline & Cytochrome C \\
\hline
\end{tabular}

$E V$, extracellular vesicle; $E L I S A$, enzyme-linked immunosorbent assay; $B C A$ assay, bicinchoninic acid assay; TSG101, tumor susceptibility gene 101

methodologic quality using criteria specific to animal studies. Items in this tool include assessments for selection bias (sequence generation, baseline characteristics, allocation concealment), performance bias (random housing, blinding), detection bias (random and blinded outcome assessment), attrition bias (completeness of outcome data), and reporting bias. Each parameter of bias for each included study will be scored as having a low, high, or unclear risk of bias. Any disagreements between the two reviewers will be resolved through discussion with a third-party member (MML).

\section{Data analysis}

Before a meta-analysis will be deemed appropriate to execute, the heterogeneity of included studies will be
Table 2 Interventional traits and dosage regimen of the extracellular vesicle therapy

\begin{tabular}{|c|c|}
\hline Question of interest & Example answers \\
\hline \multirow[t]{3}{*}{ Storage of EVs } & Frozen at $-80^{\circ} \mathrm{C}$ \\
\hline & Fresh / fridge \\
\hline & Not described \\
\hline \multirow[t]{3}{*}{ Tissue source of MSCs } & Bone marrow \\
\hline & Adipose \\
\hline & Umbilical cord/Wharton's jelly \\
\hline \multirow[t]{3}{*}{ Animal source of MSCs } & Human source \\
\hline & Mouse source \\
\hline & Rat source \\
\hline \multirow[t]{4}{*}{ EV immunocompatibility } & Xenogeneic \\
\hline & Allogeneic \\
\hline & Autologous \\
\hline & Not described \\
\hline \multirow[t]{3}{*}{ Modification of EVs } & Unmodified \\
\hline & Modified MSCs before EV isolation \\
\hline & Modified EVs directly \\
\hline \multirow[t]{4}{*}{ Route of administration } & Intravenous \\
\hline & Direct tissue injection \\
\hline & Subcutaneous \\
\hline & Intra-arterial \\
\hline \multirow[t]{3}{*}{ EV dosage units } & EV protein amount \\
\hline & EV particle number \\
\hline & EV dose based on MSC cell count \\
\hline \multirow[t]{2}{*}{ Timing of First EV dose } & Post-injury induction (treatment) \\
\hline & Pre-injury induction (prevention) \\
\hline \multirow[t]{2}{*}{ Number of doses used } & Single dose of EVs \\
\hline & Multiple doses of EVs \\
\hline
\end{tabular}

$E V$, extracellular vesicle; $M S C$, mesenchymal stromal cells

assessed. A senior author (MML, DA, DB) will review the study populations and study characteristics to judge the presence of methodologic heterogeneity. If sufficient homogeneity across studies exists, a meta-analysis will be considered appropriate to carry out. Continuous endpoints will be pooled using standardized mean differences with an inverse variance random effects method [24, 25]. Dichotomous outcomes (e.g., mortality) from each study will be pooled and described as risk ratios with 95\% confidence intervals using the DerSimonian and Laird random-effects method (Comprehensive Meta-Analysis v3.1) [26]. Statistical heterogeneity of effect sizes will be assessed using the Cochrane $I^{2}$ statistic test [27]. Thresholds for interpretation of $I^{2}$ are as follows: $0-40 \%$ (low heterogeneity), $30-60 \%$ (moderate heterogeneity), 50-90\% (substantial heterogeneity), and 75$100 \%$ (considerable heterogeneity) [27]. If there is 
Table 3 Disease domains, preclinical models, and randomization

\begin{tabular}{ll}
\hline Question of interest: & Answers \\
\hline What was the disease or condition of interest? & Brain (e.g., stroke, traumatic brain injury) \\
& Kidney (e.g., acute kidney injury, chronic kidney disease) \\
& Lung (e.g., acute lung injury, pulmonary hypertension) \\
& Bone/joint (e.g., osteoarthritis) \\
Animal model & Liver (e.g., hepatotoxicity, chronic liver disease) \\
& Cardiac (e.g., myocardial infarction) \\
Were animals randomized? & Cancer \\
& Mouse \\
& Rat \\
& Pig \\
& Yes \\
\hline
\end{tabular}

considerable heterogeneity (75-100\%), sources of heterogeneity will be explored through subgroup and sensitivity analyses.

\section{Subgroup analyses}

Planned subgroup analyses include intervention characteristics, such as tissue source of mesenchymal stromal cells (e.g., bone marrow, umbilical cord, and adipose), size of extracellular vesicles (e.g., small extracellular vesicles from 30-150 nm, or medium/large extracellular vesicles from 150-1000 nm), method of extracellular vesicle isolation (e.g., ultracentrifugation, tangential flow filtration, and size exclusion chromatography), and methods of vesicle characterization (e.g., Western Blot, electron microscopy, and flow cytometry). Additionally, studies will be subgrouped by disease domain for preclinical outcome assessment.

\section{Meta-biases assessment}

Evaluation for publication bias and selective reporting bias will be conducted using a funnel plot, generated by Comprehensive Meta-Analyst, and Egger's regression test $[28,29]$.

\section{Knowledge dissemination}

Understanding that there has been a markedly heightened interest to evaluate the therapeutic potential of mesenchymal stromal cell-derived extracellular vesicles, this review will first assess the feasibility of conducting a meta-analysis based on the final number of included preclinical studies. If a feasible number of articles are included, our team will summarize the methodology data and conduct a meta-analysis of outcomes. If the included number of articles is too large to feasibly perform multiple analyses in a single publication, a systematic review of the overall methodology being used for in vivo extracellular vesicle studies will be first published. Subsequently, analyses for each disease domain with more details (e.g., risk of bias and outcome measures) will be published as separate manuscripts in peerreviewed journals. Finally, findings will be disseminated through international conferences with basic and translational scientists to help guide future preclinical and clinical study design of extracellular vesicle therapy.

\section{Amendments}

If any amendments to this protocol are necessary, the date and specific changes to the protocol will be documented on PROSPERO with rationales as to why the alterations were required.

\section{Discussion}

Our systematic review intends to identify and summarize preclinical studies investigating the therapeutic efficacy of both small and large extracellular vesicles derived from mesenchymal stromal cells. This work updates our previous study that captured studies through 2013 [13]. Although only 17 studies were included in the previous systematic review, the therapeutic potential for this novel cell-free therapy was evident as many disease and injury states had been investigated. Since then, many more research groups around the world have begun to study extracellular vesicles, and they are now well-recognized to carry biologically active factors that mediate the therapeutic effects of mesenchymal stromal cells. Extracellular vesicles represent a novel investigative direction within regenerative medicine.

We anticipate that this comprehensive synthesis and meta-analysis of preclinical studies assessing the beneficial or adverse effects of mesenchymal stromal cellderived extracellular vesicles will generate valuable data 
to help guide future preclinical study design. More importantly, these findings can help provide considerations of future translational human participant studies.

\section{Supplementary information}

Supplementary information accompanies this paper at https://doi.org/10. 1186/s13643-019-1242-y.

Additional file 1. Preferred reporting items for systematic review and meta-analysis protocols (PRISMA-P) 2015 Checklist.

Additional file 2. Search strategy used for systematic literature search.

\section{Acknowledgements}

AT is a doctoral student supported by the Vanier Canada Graduate Scholarship with the Canadian Institutes of Health Research (CIHR). MML is supported by The Ottawa Hospital Anesthesia Alternate Funds Association. We would like to thank Risa Shorr, Librarian and Information Specialist with the Ottawa Hospital Research Institute, Ottawa, Ontario, for designing the search strategy for this systematic review.

\section{Authors' contributions}

AT, MS, DSA, and MML conceived the study design. AT, DSA, and MML were responsible for the initial drafting of the manuscript. AT and MS formulated the data extraction questions and tables. DJS, DAF, JM, and DB provided important revisions for the protocol development as well as data extraction questions. MML is the guarantor of this review. All authors were involved in manuscript revisions before final approval.

\section{Funding}

This work received no external or internal sources of funding.

\section{Availability of data and materials}

Not applicable.

\section{Ethics approval and consent to participate}

Not applicable.

\section{Consent for publication}

Not applicable.

\section{Competing interests}

DJS is President and CEO of Northern Therapeutics (Montreal, QC, Canada).

The remaining authors have no competing interests to declare.

\section{Author details}

${ }^{1}$ Department of Cellular and Molecular Medicine, University of Ottawa, Ottawa, Canada. ${ }^{2}$ Clinical Epidemiology Program, BLUEPRINT Translational Research Group, Ottawa Hospital Research Institute, Ottawa, Canada. ${ }^{3}$ Regenerative Medicine Program, Ottawa Hospital Research Institute, Ottawa, Canada. ${ }^{4}$ Department of Medicine, University of Ottawa, The Ottawa Hospital, Ottawa, Canada. ${ }^{5}$ Department of Surgery, University of Ottawa, The Ottawa Hospital, Ottawa, Canada. 'Department of Nephrology, University of Ottawa, The Ottawa Hospital, Ottawa, Canada. ${ }^{7}$ Kidney Research Centre, Ottawa Hospital Research Institute, Ottawa, Canada. ${ }^{8}$ Learning Services, The Ottawa Hospital, Ottawa, Canada. ${ }^{9}$ Department of Anesthesiology and Pain Medicine, University of Ottawa, The Ottawa Hospital, Ottawa, Canada.

Received: 20 March 2019 Accepted: 24 November 2019 Published online: 12 December 2019

\section{References}

1. Kern S, Eichler H, Stoeve J, Klüter H, Bieback K. Comparative analysis of mesenchymal stem cells from bone marrow, umbilical cord blood, or adipose tissue. Stem Cells. 2006;24(5):1294-301.

2. Jung Y, Bauer G, Nolta JA. Concise review: induced pluripotent stem cellderived mesenchymal stem cells: progress toward safe clinical products. Stem Cells. 2012;30(1):42-7.
3. Squillaro T, Peluso G, Galderisi U. Clinical trials with mesenchymal stem Cells: an update. Cell Transplant. 2016;25(5):829-48.

4. Mclntyre LA, Stewart DJ, Mei SH, et al. Cellular immunotherapy for septic shock. A phase I clinical trial. Am J Respir Crit Care Med. 2018; 197(3):337-47.

5. Matthay MA, Calfee CS, Zhuo H, et al. Treatment with allogeneic mesenchymal stromal cells for moderate to severe acute respiratory distress syndrome (START study): a randomised phase 2a safety trial. Lancet Respir Med. 2018.

6. Lalu MM, Mazzarello S, Zlepnig J, et al. Safety and efficacy of adult stem cell therapy for acute myocardial infarction and ischemic heart failure (SafeCell Heart): a systematic review and meta-analysis. Stem Cells Transl Med. 2018; 7(12):857-66.

7. Gao F, Chiu S, Motan D, et al. Mesenchymal stem cells and immunomodulation: current status and future prospects. Cell Death Dis. 2017;7(1):e2062

8. Galipeau J, Sensebe L. Mesenchymal stromal cells: clinical challenges and therapeutic opportunities. Cell stem cell. 2018;22(6):824-33.

9. Prockop DJ, Brenner M, Fibbe WE, et al. Defining the risks of mesenchymal stromal cell therapy. Cytotherapy. 2010;12(5):576-8.

10. György B, Szabó TG, Pásztói M, et al. Membrane vesicles, current state-ofthe-art: emerging role of extracellular vesicles. Cell Mol Life Sci. 2011;68(16): 2667-88.

11. Yeo RW, Lai RC, Zhang B, et al. Mesenchymal stem cell: an efficient mass producer of exosomes for drug delivery. Advanced drug delivery reviews. 2013:65(3):336-41.

12. Katsuda T, Kosaka N, Takeshita F, Ochiya T. The therapeutic potential of mesenchymal stem cell-derived extracellular vesicles. Proteomics. 2013; 13(10-11):1637-53.

13. Akyurekli C, Le Y, Richardson RB, Fergusson D, Tay J, Allan DS. A systematic review of preclinical studies on the therapeutic potential of mesenchymal stromal cell-derived microvesicles. Stem Cell Rev Rep. 2015:11(1):150-60.

14. Taljaard M, Ward MR, Kutryk MJ, et al. Rationale and design of enhanced angiogenic cell therapy in acute myocardial infarction (ENACT-AMI): the first randomized placebo-controlled trial of enhanced progenitor cell therapy for acute myocardial infarction. Am Heart J. 2010;159(3):354-60.

15. Granton J, Langleben D, Kutryk MB, et al. Endothelial NO-synthase geneenhanced progenitor cell therapy for pulmonary arterial hypertension: The PHACeT trial. Circ Res. 2015;117(7):645-54.

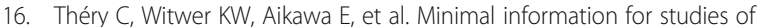
extracellular vesicles 2018 (MISEV2018): a position statement of the International Society for Extracellular Vesicles and update of the MISEV2014 guidelines. J Extracell Vesicles. 2018;7(1):1535750.

17. Shamseer $L$, Moher $D$, Clarke $M$, et al. Preferred reporting items for systematic review and meta-analysis protocols (PRISMA-P) 2015: elaboration and explanation. BMJ. 2015;349:97647.

18. McGowan J, Sampson M, Lefebvre C. An evidence based checklist for the peer review of electronic search strategies (PRESS EBC). Evidence Based Library and Information Practice. 2010:5(1):149-54.

19. Sampson M, McGowan J, Cogo E, Grimshaw J, Moher D, Lefebvre C. An evidence-based practice guideline for the peer review of electronic search strategies. J Clin Epidemiol. 2009;62(9):944-52.

20. de Vries RB, Hooijmans CR, Tillema A, Leenaars M, Ritskes-Hoitinga M. A search filter for increasing the retrieval of animal studies in Embase. Lab Anim. 2011;45(4):268-70.

21. Hooijmans CR, Tillema A, Leenaars M, Ritskes-Hoitinga M. Enhancing search efficiency by means of a search filter for finding all studies on animal experimentation in PubMed. Lab Anim. 2010;44(3):170-5.

22. Leenaars $M$, Hooijmans $C R$, van Veggel $N$, et al. A step-by-step guide to systematically identify all relevant animal studies. Lab Anim. 2012; 46(1):24-31.

23. Hooijmans CR, Rovers MM, de Vries RB, Leenaars M, Ritskes-Hoitinga M, Langendam MW. SYRCLE's risk of bias tool for animal studies. BMC Med Res Methodol. 2014:14(1):43.

24. DerSimonian R, Laird N. Meta-analysis in clinical trials. Control Clin Trials. 1986:7(3):177-88

25. Friedrich JO, Adhikari NK, Beyene J. The ratio of means method as an alternative to mean differences for analyzing continuous outcome variables in meta-analysis: a simulation study. BMC Med Res Methodol. 2008;8(1):32.

26. Collaboration C. Review Manager (RevMan) version 5.3. Copenhagen: The Nordic Cochrane Centre. In:2014. 
27. Higgins JPT AD, Sterne JAC, editors. Cochrane handbook for systematic reviews of interventions Available from: http://handbook-5-

1 cochraneorg/. 2011.

28. Sterne JA, Sutton AJ, loannidis JP, et al. Recommendations for examining and interpreting funnel plot asymmetry in meta-analyses of randomised controlled trials. BMJ. 2011;343:d4002.

29. Egger M, Smith GD, Schneider M, Minder C. Bias in meta-analysis detected by a simple, graphical test. BMJ. 1997;315(7109):629-34.

\section{Publisher's Note}

Springer Nature remains neutral with regard to jurisdictional claims in published maps and institutional affiliations.

Ready to submit your research? Choose BMC and benefit from:

- fast, convenient online submission

- thorough peer review by experienced researchers in your field

- rapid publication on acceptance

- support for research data, including large and complex data types

- gold Open Access which fosters wider collaboration and increased citations

- maximum visibility for your research: over $100 \mathrm{M}$ website views per year

At BMC, research is always in progress.

Learn more biomedcentral.com/submissions 